\title{
Improved constraints on Neptune's atmosphere from submillimetre-wavelength observations
}

\author{
A. Marten ${ }^{1}$, H. E. Matthews ${ }^{2, \star}$, T. Owen ${ }^{3}$, R. Moreno ${ }^{1}$, T. Hidayat ${ }^{4}$, and Y. Biraud ${ }^{1}$ \\ 1 LESIA, Observatoire de Paris-Meudon, 92195 Meudon, France \\ e-mail: andre.marten@obspm.fr \\ 2 Joint Astronomy Centre, Hilo, HI 96720, USA \\ 3 Institute for Astronomy, University of Hawaii, Honolulu, HI 96822, USA \\ ${ }^{4}$ Department of Astronomy and Bosscha Observatory, Bandung, 40132, Indonesia
}

Received 20 July 2004 / Accepted 14 September 2004

\begin{abstract}
We describe observations of Neptune carried out using the James Clerk Maxwell Telescope (Mauna Kea, Hawaii) during September 1998. High-quality data for the sub-mm-wavelength rotational transitions of HCN $(J=4-3)$ and CO $(J=$ 3-2 and 4-3) were obtained, and we use the data to make improved determinations of the abundances of these trace species and the temperature profile of Neptune's atmosphere.
\end{abstract}

Key words. planets and satellites: individual: Neptune - radio lines: solar system

\section{Introduction}

Ground-state rotational lines of $\mathrm{HCN}$ and $\mathrm{CO}$ were first observed from the atmosphere of Neptune in 1991 (Marten et al. 1991). Subsequent observational campaigns conducted at millimetre $(\mathrm{mm})$ and submillimetre (submm) wavelengths at the JCMT (James Clerk Maxwell Telescope), CSO (Caltech Submillimeter Observatory), both on Mauna Kea, Hawaii, and with the IRAM telescopes (Institut de Radioastronomie Millimétrique, Granada, Spain and Plateau de Bure, France) have since led to models of the vertical distributions of both species. Various spectroscopic techniques have been used to measure the relatively narrow $(\sim 20 \mathrm{MHz})$ emission lines from the upper atmosphere (Rosenqvist et al. 1992; Marten et al. 1993a; Lellouch et al. 1994). Absorption has been observed only for CO (Guilloteau et al. 1993; Naylor et al. 1994), where the line profiles are quite broad, typically $20 \mathrm{GHz}$ at $345 \mathrm{GHz}$. $\mathrm{HCN}$ condenses in the troposphere and thus cannot exhibit absorption features. Additional CO measurements at UV wavelengths have been obtained by Courtin et al. (1996), using the Hubble Space Telescope.

Broadly, the measurements show that CO is (a) uniformly distributed in Neptune's upper atmosphere with a mixing ratio of about $1 \mathrm{ppm}$ and (b) that the stratospheric HCN mixing ratio is about $1 \mathrm{ppb}$. However, the vertical profile of $\mathrm{HCN}$ is not well constrained by the published data in the upper stratosphere above the 1.0-mbar pressure level; here we report on improved observational data.

^ Present address: National Research Council of Canada, DRAO, Penticton, BC V2A 6J9, Canada.
The formation of $\mathrm{CO}$ and $\mathrm{HCN}$ was first explored by Marten et al. (1993a, Paper I, hereafter), who inferred that CO was present throughout the atmosphere of Neptune, whereas HCN could exist only in the stratosphere. A new thermochemical model of Neptune was subsequently proposed by Lodders \& Fegley (1994) to explain the high abundance of CO; this in turn implied a very large $\mathrm{O} / \mathrm{H}$ ratio in the interior of the planet. Further, Lellouch et al. (1994) have presented a detailed analysis of the formation of $\mathrm{HCN}$ in the Neptunian stratosphere. Since HCN must originate from the reaction between $\mathrm{CH}_{4}$ (probably through $\mathrm{CH}_{3}$ radicals resulting from dissociation of the latter) and $\mathrm{N}$ atoms, two incident sources of $\mathrm{N}$ atoms (internal and external to Neptune) were investigated: galactic cosmic ray (GCR) impacts with $\mathrm{N}_{2}$ in Neptune's atmosphere, and incident $\mathrm{N}$ atoms originating from Triton. Although the latter hypothesis was attractive, the former was favoured by Paper I, while Lellouch et al. (1994) concluded that both formation mechanisms are viable sources of $\mathrm{N}$ atoms.

In an attempt to better understand the origin of $\mathrm{HCN}$ on Neptune we undertook a renewed campaign of sensitive submm-wavelength observations of $\mathrm{HCN}$ and $\mathrm{CO}$ towards the planet during September 1998. Our previous analysis in 1991 was limited by the rather low signal-to-noise ratio obtained at that time; hence our goal for the renewed work was to obtain well-calibrated spectra with both high spectral resolution and a very low noise level. This was facilitated both by the improved performance of receivers then newly available at the JCMT, and by long integration times obtained during exceptional ("El Niño") observing conditions. To obtain a reliable absolute flux calibration scale concomitant measurements of 
Table 1. Summary of heterodyne observations of Neptune.

\begin{tabular}{|c|c|c|c|c|c|c|c|c|c|c|}
\hline Line & $\begin{array}{l}\text { Frequency } \\
\quad(\mathrm{GHz})\end{array}$ & $\begin{array}{l}\text { Date } \\
\text { of observations }\end{array}$ & Telescope & $\begin{array}{c}T_{\mathrm{rec}}^{\mathrm{DSB}} \\
(\mathrm{K})\end{array}$ & $\begin{array}{l}T_{\text {sys }} \\
(\mathrm{K})\end{array}$ & $\begin{array}{c}T_{\mathrm{int}} \\
(\mathrm{min})\end{array}$ & $\begin{array}{l}\text { Resolution } \\
\quad(\mathrm{kHz})\end{array}$ & $\begin{array}{c}B_{\mathrm{w}} \\
(\operatorname{arcsec})\end{array}$ & $B_{\text {eff }}$ & Reference \\
\hline \multirow[t]{4}{*}{$\mathrm{HCN}(4-3)$} & 354.505 & June 1995 & $\mathrm{CSO}$ & 200 & 670 & 85 & 100,570 & 22.0 & 0.62 & $\mathrm{~A}$ \\
\hline & & June 1995 & JCMT & 250 & 1300 & 110 & 950 & 14.5 & 0.54 & A \\
\hline & & June 1996 & JCMT & 240 & 2400 & 50 & 650 & 14.5 & 0.54 & \\
\hline & & September 1998 & JCMT & 210 & 450 & 100 & 378 & 14.5 & 0.54 & $\mathrm{C}$ \\
\hline \multirow[t]{3}{*}{$\mathrm{CO}(4-3)$} & 461.040 & June 1993 & $\mathrm{CSO}$ & 260 & 3100 & 34 & 570 & 17.0 & 0.55 & B \\
\hline & & June 1993 & JCMT & 250 & 2300 & 30 & 745 & 12.0 & 0.40 & $\mathrm{~B}$ \\
\hline & & September 1998 & JCMT & 320 & 1300 & 110 & 189,756 & 11.5 & 0.40 & $\mathrm{C}$ \\
\hline $\mathrm{CO}(3-2)$ & 345.796 & September 1998 & JCMT & 210 & 380 & 30 & 756 & 14.5 & 0.54 & $\mathrm{C}$ \\
\hline $\mathrm{CO}(2-1)$ & 230.538 & November 1995 & IRAM & 140 & 380 & 25 & 100,1000 & 10.4 & 0.40 & $\mathrm{C}$ \\
\hline
\end{tabular}

Notes:

- Observations of the $\mathrm{HCN}(4-3), \mathrm{CO}(2-1)$ and $\mathrm{CO}(3-2)$ transitions were obtained for the first time in 1991 with both the JCMT and CSO telescopes (Paper I). All subsequent measurements are presented in this table.

- All telescopic parameters are defined in Sect. 3.

- Values of the sub-Earth latitude vary by less than $1.5^{\circ}$ between 1993 and 1998, (-26.6 ${ }^{\circ}$ in September 1998).

- A: Marten et al. (1995); B: Marten et al. (1993b); C: this work.

Uranus were performed. Note that, despite several efforts, no molecular emission or absorption features have been observed in the $\mathrm{mm} / \mathrm{submm}$-wave spectrum of Uranus (Rosenqvist et al. 1992; Guilloteau et al. 1993; Paper I). Finally, since careful synthetic computations are clearly needed in such flux comparisons an updated radiative transfer code was developed, based on that employed for the interpretation of microwave spectra of Titan and Jupiter (see for instance Hidayat et al. 1997; Moreno et al. 2001).

We first present our new synthetic model calculations in detail in Sect. 2. The observations and data reduction are described next in Sect. 3. We then compare the observed spectra with equivalent synthetic spectra in Sects. 4 and 5, and finally we give a general synopsis of our results in Sect. 6 .

\section{Radiative transfer modeling in the submm-wavelength range}

\subsection{Geometric considerations}

Radiative transfer modeling usually assumes a plane-parallel geometry in which, for example, the thickness of the contributing atmosphere is relatively small compared to the mean planetary radius. This simplification was adequate for our early investigations of Neptune's disk-averaged spectra. However, for our observations of Titan's extended atmosphere this approach was no longer valid, and a more realistic code was developed that takes into account the spherical geometry of the planet and includes limb brightening effects (Hidayat et al. 1997). We here adapt this technique to the investigation of Neptune. As such simulations must be able to model highvelocity-resolution spectra, Doppler shifts due to the planetary rotation (2.66 $\mathrm{km} \mathrm{s}^{-1}$ at the equatorial limbs for Neptune) become significant and must also be taken into account. This feature, as well as the convolution of the emitted flux density with the Gaussian beam of the telescope, were introduced in the radiative transfer algorithm, as discussed by Moreno et al. (2001). While the former calculation introduces a spectral smoothing process of the emission line features, the latter precisely evaluates the beam-weighted flux emitted at the limbs. (Note that a simplified formulation of this second aspect is generally used when planets of small size compared to the telescope beam width are observed; see Sect. 4.)

While the code however considers the giant planets as perfect spheres, it does provide sufficiently accurate computations of line shape profiles and emitted fluxes at all altitudes probed in the atmosphere. As previously noted by Moreno et al. (2001), simulations of Jupiter's atmosphere within the same wavelength domain with a similar computation code yield errors of a few percent compared to those using an oblate spheroid geometry for the planetary atmosphere. We may expect smaller errors for Uranus and Neptune, neither of which, unlike Jupiter, are resolved by the telescope beam (see Sect. 3 and Table 1). They have also respective ratios of equatorial/polar radius smaller than that for Jupiter. We note that brightness temperature differences of less than $4 \%$ are apparent between our first simulations, presented in Paper I, and those shown here (see Figs. 2 and 3 below, and Sect. 4). Our present spectral simulations represent a further refinement beyond those proposed by Lellouch et al. (1994) in a reanalysis of our $1991 \mathrm{HCN}$ data.

\subsection{Opacity}

The basic inputs to the line-by-line radiative transfer simulations are the abundances (and hence opacities) of all atmospheric constituents, and the temperature profile. In the submm-wavelength range, continuum opacities in the atmospheres of Uranus and Neptune result mainly from 


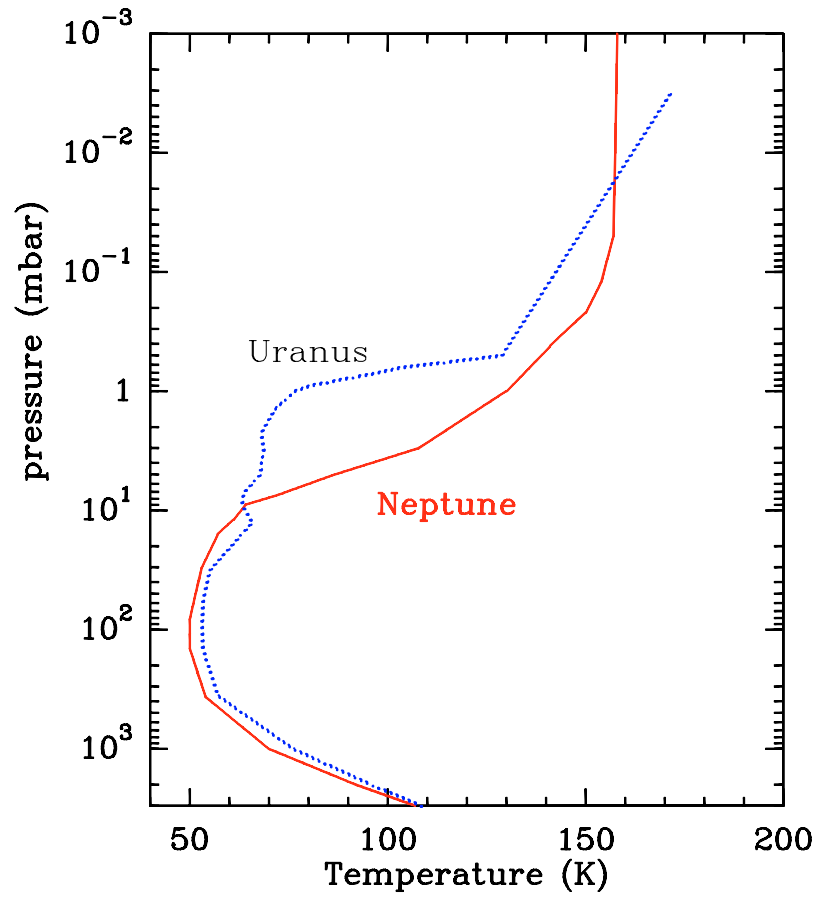

Fig. 1. Nominal disk-averaged temperature profiles for Neptune (solid line) and Uranus (dotted line) used in a preliminary analysis. This can be compared with the results of our renewed work in Fig. 5.

pressure-induced absorptions due to collisions of $\mathrm{H}_{2}$ with $\mathrm{H}_{2}$, $\mathrm{He}$ and $\mathrm{CH}_{4}$. We computed the absorption coefficients for these interactions using the codes developed by Borysow \& Frommhold (1986) and Birnbaum et al. (1996). We also considered potential contributions due to $\mathrm{NH}_{3}, \mathrm{PH}_{3}$, and $\mathrm{H}_{2} \mathrm{~S}$, as advocated by DeBoer \& Steffes (1996). As for our work with Jupiter (Moreno et al. 2001) we used a Van-Vleck and Weisskopf lineshape profile. It is worth noting that the use of another formalism such as that of Ben-Reuven does not change significantly the global molecular opacity in the submmwavelength range. A Voigt profile lineshape was assumed for $\mathrm{CO}$ and $\mathrm{HCN}$ lines with $\mathrm{H}_{2}$ collision-broadened half-widths of 3 and $6 \mathrm{MHz}$ /Torr at $T=300 \mathrm{~K}$, as in Paper I (Draegert \& Williams 1968; Rohart et al. 1987) ${ }^{1}$. Since the central region of the emission-line cores are expected to be relatively narrow, a variable frequency step as low as $10 \mathrm{kHz}$ was automatically selected to adequately calculate the line profiles near their centres. Spectroscopic data used in this paper were taken from the JPL molecular line catalog (Pickett et al. 1998).

\subsection{Atmospheric models of Uranus and Neptune}

The baseline temperature profiles of both planets plotted in Fig. 1 are very similar to the disk-averaged profiles we used previously and, in the upper tropospheres and lower stratospheres, consistent with the equatorial structures determined by Flasar et al. (1987), Conrath et al. (1991, 1998), from Voyager IRIS spectra. For Uranus, the profile (from Lindal 1992) may

${ }_{1}$ The $\mathrm{CO}-\mathrm{H}_{2}$ pressure broadening parameter agrees well with the values recently measured by Mengel et al. (2000) in the $J=1-0$ and $J=2-1$ millimetre-wavelength transitions. differ from the modeling of Marley \& McKay (1999), since our present study is concerned essentially with the mean tropospheric profile on this planet. We nevertheless considered that deviations of typically $\pm 2 \mathrm{~K}$ around this profile could exist in the troposphere, such as can be seen in models presented by the latter authors. For Neptune, the nominal thermal model is based on Lindal (1992); Bézard et al. (1991, 1999), and also agrees in the $1-\mu$ bar region with determinations by Roques et al. (1994) from stellar occultation observations. We also considered the model profiles of Uranus and Neptune used by Feuchtgruber et al. (1999) in the analysis of infrared ISO spectra. Several possible thermal models of Neptune are therefore discussed below in Sect. 5.

We assume that all trace constituents mentioned in Sect. 2.2, with the exception of $\mathrm{CO}$, condense in the troposphere at pressure levels $>1$ bar $\left(\mathrm{NH}_{3}\right.$ and $\mathrm{H}_{2} \mathrm{~S}$ nominally condense at much deeper levels). As the continuum emission seen in both planets is formed in the troposphere around 1 bar (see the contribution functions computed near $350 \mathrm{GHz}$ in Paper I), we note that the submm-wavelength range between 350 and $500 \mathrm{GHz}$ is quite insensitive to additional opacities due to $\mathrm{NH}_{3}$, $\mathrm{PH}_{3}$ and $\mathrm{H}_{2} \mathrm{~S}$. The same conclusions can be drawn from the work of DeBoer \& Steffes (1996). In the mm-wavelength range, between 190 and $310 \mathrm{GHz}$, Encrenaz et al. (1996) did not detect any absorption features towards either Uranus or Neptune, implying in particular very low abundance for phosphine.

It follows that the only saturation law that needs to be known with precision is that of HCN. Here we adopt the most recent vapour pressure law (given by Lellouch et al. 1994), in the form:

$\log _{10} V_{\mathrm{p}}(\mathrm{mbar})=11.535-\frac{2318}{T(\mathrm{~K})}$.

For our purposes we may first assume that $\mathrm{HCN}$ is uniformly mixed above a condensation level near 3 mbar.

The $\mathrm{H}_{2} / \mathrm{He}$ ratio assuming ortho-para $\mathrm{H}_{2}$ abundance consistent with the thermal equilibrium and the $\mathrm{CH}_{4}$ abundance adopted for Uranus and Neptune are those used in Paper I. The $\mathrm{H}_{2} / \mathrm{He}$ ratio we used previously, and derived from IRIS and radio experiment data of Voyager 2, have since been confirmed from ISO infrared spectra (Burgdof et al. 2003). Other physical parameters, such as equatorial and polar radii (at the 1-bar pressure level) and the accelerations of gravity, are those determined by Lindal (1992) from a complete analysis of radio occultation data obtained with the Voyager 2 spacecraft.

\subsection{Examples of low resolution spectra}

Figure 2 presents synthetic low-resolution spectra of Uranus and Neptune between 200 and $500 \mathrm{GHz}$. For simplicity, the spectra are shown on a brightness temperature $T_{\mathrm{B}}$ (Kelvin) scale rather than flux density. Because neither planet is resolved by the primary beam of the telescope used to obtain these data, the $T_{\mathrm{B}}$ scale employed here, as in Paper I, is equivalent to a mean brightness temperature averaged over the planetary disk, as described below in Sects. 3 and 4. For comparison we include in the figure broad-band bolometric measurements 


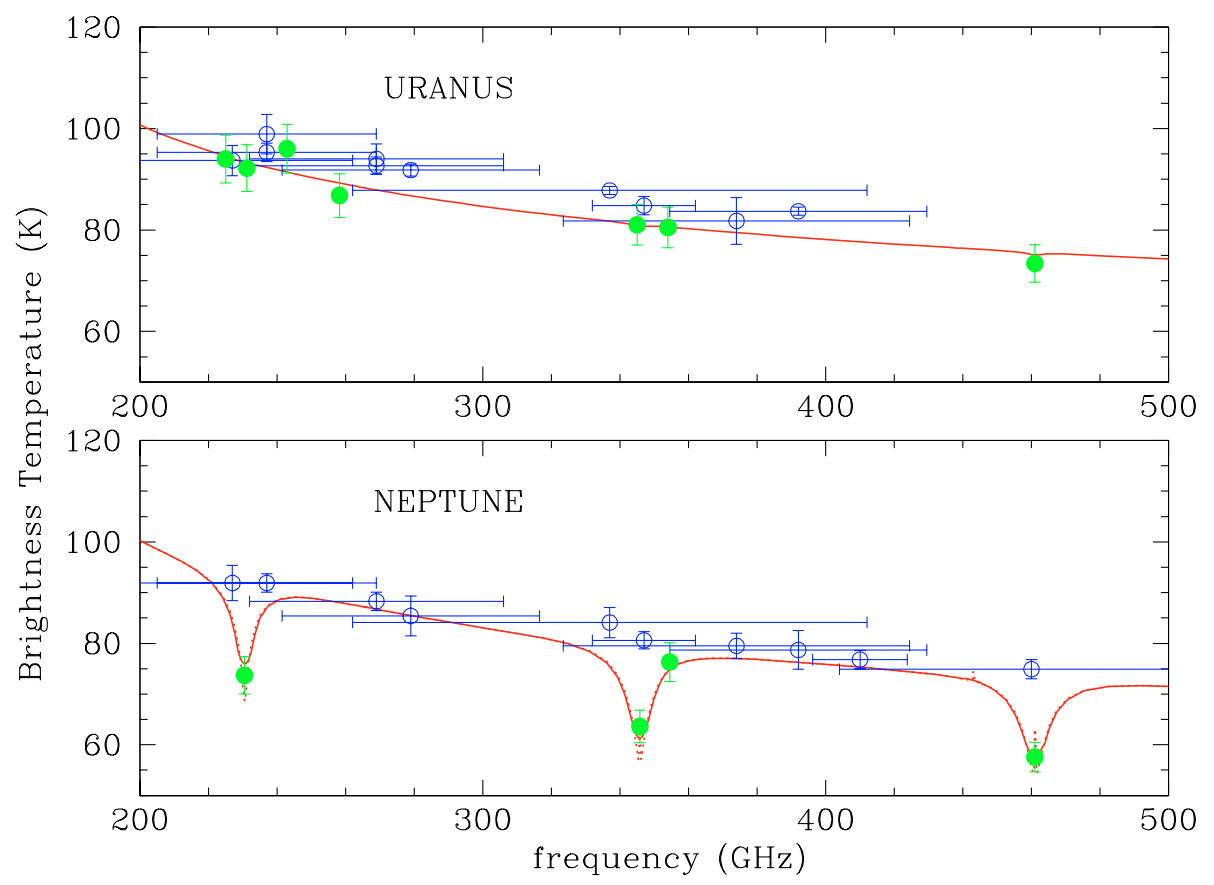

Fig. 2. Comparison of available measurements with atmospheric modeling. The continuous lines show examples of synthetic spectra of Uranus (top panel) and Neptune (bottom panel) computed with a spectral resolution of $5.0 \mathrm{GHz}$. For Neptune we show also spectra computed for a $0.5-\mathrm{GHz}$ resolution (dotted line) for comparison with our present measurements; the effect of improved spectral resolution is clearly seen at the line cores. The thermal profiles shown in Fig. 1 have been used in these calculations. A CO mole fraction for Neptune of 1 ppm has been assumed. For comparison, broad-band bolometer measurements obtained by Griffin \& Orton (1993) are shown as open circles, where the horizontal bars indicate the photometric bandwidths, while the filled circles show our heterodyne measurements averaged over the $0.5-\mathrm{GHz}$ bandwidth obtained with the JCMT and IRAM-30 m telescopes. Notice that very little brightness temperature differences may exist between low-resolution $(5-\mathrm{GHz})$ calculations and bolometer data since the spectral bandwidths of the latter measurements are much larger than $5 \mathrm{GHz}$.

acquired by Griffin \& Orton (1993) and our $0.5-\mathrm{GHz}-$ bandwidth-averaged heterodyne measurements obtained during our observing sessions (see Sect. 3). Although the spectral bandwidths of the bolometric data points are much broader than the 5.0-GHz resolution of the theoretical spectra, there is good agreement with the calculations, and also with our continuum measurements of Uranus and Neptune.

\section{Heterodyne submm-wave observations}

The observations carried out in this program subsequent to the early data obtained in 1991 are summarized in Table 1. Observations of the $\mathrm{HCN}$ ground-state rotational transition $J=4-3$ at $354 \mathrm{GHz}$ were made in June 1995 using both the CSO and the JCMT (Marten et al. 1995). Subsequent observations in June 1996 were performed in rather poor weather conditions. The first useful measurements of CO $(J=4-3)$ in emission (at $461 \mathrm{GHz}$ ) were obtained at the JCMT and the CSO in June 1993 (Marten et al. 1993b) and subsequently in June 1995 at the JCMT.

In September 1998, two new receivers operating at the JCMT in the 315-370 and 450-505 GHz regions, coincidentally coupled with excellent weather conditions, allowed very sensitive, well-calibrated measurements of key rotational lines of CO ( $J=3-2$ and 4-3) and $\mathrm{HCN}(J=4-3)$ to be made for the first time in this programme. The observational log in Table 1 summarizes the receiver parameters and other details. Both receivers operate in single-sideband (SSB) mode and with two SIS mixers simultaneously detecting orthogonallypolarized radiation. The same observing periods were also devoted to extensive Jupiter observations, as described by Matthews et al. (2002), and the continuum measurements of Uranus and Neptune mentioned here were used for verifying the calibration of the Jupiter spectra taken with the $345-\mathrm{GHz}$ receiver. These continuum data are incorporated in Fig. 2.

We report in detail here on these last spectral measurements of CO and HCN, taken on 14-18 September 1998. Calibration procedures as well as telescope pointing and focus adjustment were those usually used at the JCMT. Frequency setup and flux calibration precision were systematically verified against standard astronomical sources such as G34.3 or NGC 6334, and also against Jupiter (and Saturn, for the continuum) ${ }^{2}$. The latter verification was particularly important in the present case, since we needed to determine the absolute fluxes of Uranus and Neptune with a precision better than $10 \%$. The values of the half-power beam width $\left(B_{\mathrm{w}}\right)$, given as 14.5 and 11.5 arcsec for the 345 and $461-\mathrm{GHz}$ bands, respectively, were regularly verified by observing Uranus. The corresponding main beam efficiencies $\left(B_{\text {eff }}\right)$ of the telescope are 0.54 and 0.40 , respectively.

An examination of measured and theoretical results displayed in Fig. 2 will show that the errors affecting our

\footnotetext{
${ }^{2}$ Broad-band or low-spectral resolution measurements of Jupiter and Saturn reported by Griffin et al. (1986), Weisstein \& Serabyn (1996), Goldin et al. (1997) yield useful constraints on absolute brightness temperatures of planets in the submm-wavelength range.
} 


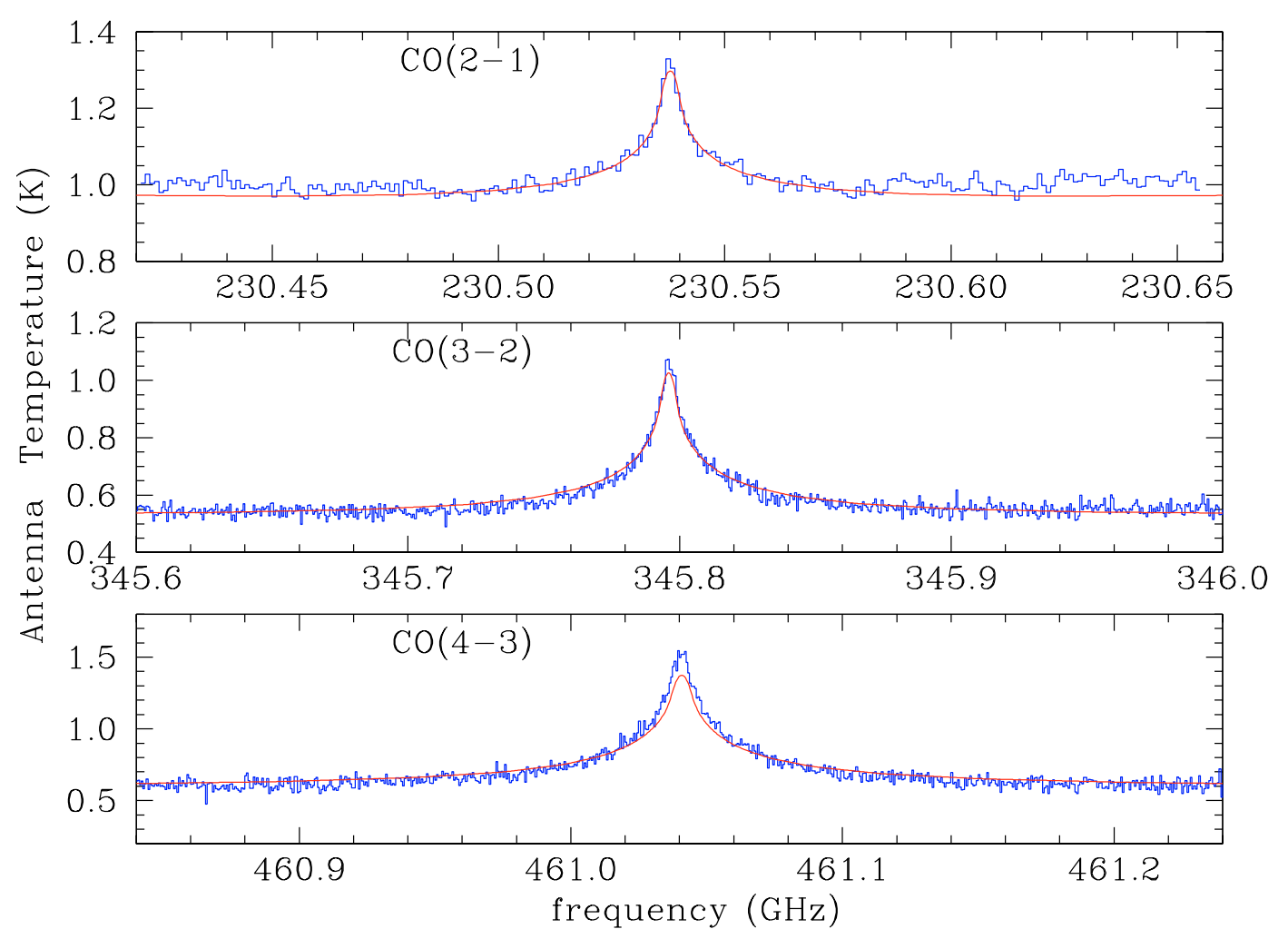

Fig. 3. Spectra of Neptune observed in rotational transitions of CO at 230, 345 and $461 \mathrm{GHz}$, (from top to bottom). In all three cases a beam-switched technique was used. Observational details for the spectra are given in Table 1. The 230-GHz line was observed with the IRAM 30-m telescope in November 1995. The two other lines were obtained with the JCMT in September 1998. Superposed on each spectrum are synthetic calculations (solid line) using the thermal profile shown in Fig. 1 and a CO mixing ratio of 1 ppm. Some discrepancies between the model and the observations can be seen; these are considered in Sect. 5.

continuum data are $<5 \%$, as compared to an upper limit of $10 \%$ being expected for our measurements. In particular, it should be noted that all spectral datasets recorded since 1993, using both the JCMT and the CSO, are internally very consistent, so that the line spectra used in the present work appear to be placed on a well-calibrated intensity scale.

In Sect. 4 we present an additional measurement of the $\mathrm{CO}(J=2-1)$ line towards Neptune obtained in November 1995 with the IRAM 30-m telescope. This observation was obtained to compare our submm-wavelength data with mm-wavelength spectra taken at a lower frequency with another telescope, as was also done previously in Paper I. From the model simulations given in Fig. 3 one can see that this $\mathrm{mm}$-wave $\mathrm{CO}$ spectrum also agrees very well with the submmwavelength data for a single value for the abundance of CO.

For all the 1998 JCMT observations we used a standard beam-switched procedure with a beam throw of 60 arcsec in azimuth. The mean SSB system temperatures $\left(T_{\text {sys }}\right)$ were about 380,450 and $1300 \mathrm{~K}$ at 345,354 and $461 \mathrm{GHz}$ respectively. Exceptionally low noise temperatures were obtained on this occasion, due to the excellent weather conditions as noted previously, characterized by a zenith optical depth at $225 \mathrm{GHz}$ routinely lower than 0.05 . The fact that the present data are of much higher quality than those obtained in 1991 and 1995 , can be attributed to a fortuitous combination of stable, transparent sky conditions, much improved receiver performance overall, and also to the fact that the observations were limited to higher elevations, above $40^{\circ}$. These attributes also resulted in consistently good calibration and the absence of spurious baseline irregularities in the spectra. The spectral resolution of the raw data was either 189 or $378 \mathrm{kHz}$, depending on the spectrometer configuration. The two independent polarisation outputs from the receivers were coadded after checking that the individual signals were closely similar. Cycles of 10-min integrations were interwoven with frequent hot/cold load calibrations, and subsequently combined to form single averaged spectra. Observations taken on separate days were put together in post-processing by allowing for the differential velocity shift of Neptune with respect to the Earth from day to day. Typical final integration times ranged from 0.5 to $2 \mathrm{~h}$ (see Table 1). Our final spectra of HCN and CO are shown in Figs. 3 and 4, where we compare them with synthetic spectra derived from models of Neptune's atmosphere, as discussed in the next section.

\section{Comparison of the observations with synthetic spectra}

Any comparison of synthetic spectra with observations requires that the antenna characteristics be known to a compatible degree. The observations result directly in spectra calibrated in antenna temperature corrected for extinction in the Earth's atmosphere $\left(T_{\mathrm{a}}^{*}\right)$. The computation models yield the disk-averaged flux density spectra for the planet, which can then be transformed into mean brightness temperature $\left(T_{\mathrm{B}}\right)$ 


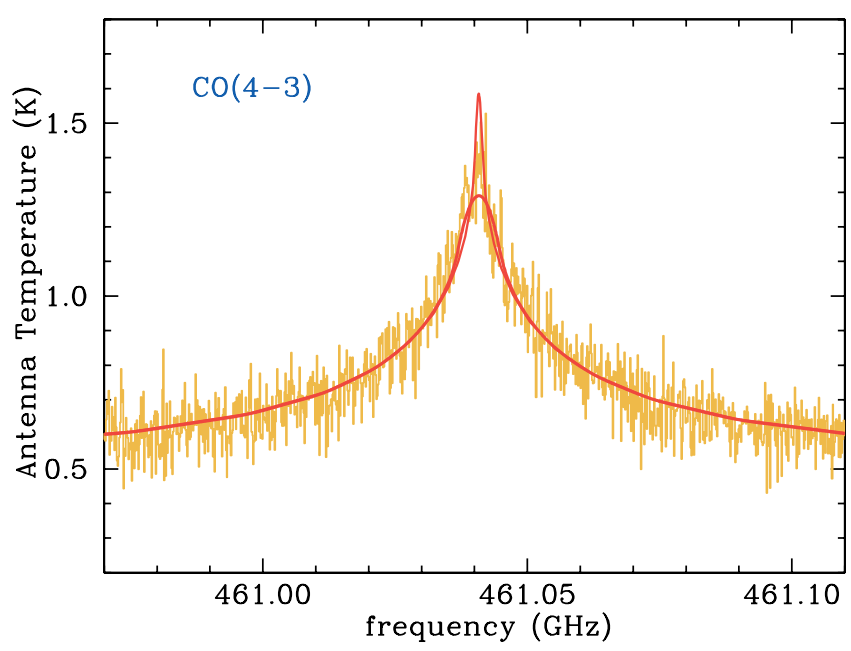

Fig. 4. Antenna temperature spectrum of Neptune observed in the rotational transition $J=4-3$ of CO. The spectral resolution is $189 \mathrm{kHz}$ and the total integration time $110 \mathrm{~min}$. Here we display only the central $140 \mathrm{MHz}$ of the spectrum; cf. Fig. 3 (bottom panel). Superposed are the results of two calculations carried out for the same spectral resolution, but considering (thick line) or neglecting (thin line) the rotational velocity of Neptune. In the former calculation the value of the sub-Earth latitude at Neptune of $-26.6^{\circ}$ was adopted (see Table 1). Differences can be seen around the line centre. Nominal profiles of Fig. 5 are used. This example illustrates a limitation of full-disk measurements in the retrieval of physical parameters in the upper atmosphere above 0.01 mbar.

spectra. In Paper I we assumed the telescope beam to be Gaussian (and characterized by the full beamwidth at halfpower $B_{\mathrm{w}}$ ), which allowed us to use the usual simple relationship between $T_{\mathrm{a}}^{*}$ and $T_{\mathrm{B}}$ :

$T_{\mathrm{B}}=\frac{T_{\mathrm{a}}^{*}}{B_{\mathrm{eff}}\left(1-2^{-\left(\theta / B_{\mathrm{w}}\right)^{2}}\right)}$.

Here $\theta$ is the planetary apparent angular size derived from the geocentric distance of the planet at the epoch of the observation and its mean diameter. The planetary 1-bar pressure level is often utilized for the calculation of $\theta$, as in Fig. 2, in which a comparison with broad-band measurements is shown.

In the interests of precision (see Sect. 2.1) the telescope beam-profile and the planetary size $\theta_{\mathrm{s}}$ including an extended atmosphere were directly incorporated into the computations, so that the above relationship is no longer used. The equatorial radius has been assumed for Neptune since the south pole was Earth-facing, while the polar radius was adopted for Uranus. Values of $\theta_{\mathrm{s}}$ considered for the September 1998 observations are 3.73 and 2.37 arcsec for Uranus and Neptune, respectively. The JCMT parameters $B_{\text {eff }}$ and $B_{\mathrm{w}}$ have been given in Sect. 3 for the frequencies of our observations. The CSO parameters recommended for the 1995 data near $345 \mathrm{GHz}$ (Wang et al. 1994) were $B_{\text {eff }}=0.62$ and $B_{\mathrm{w}}=22$ arcsec. For the IRAM observations at $230 \mathrm{GHz}$, we have adopted $B_{\text {eff }}=0.40$ and $B_{\mathrm{w}}=10.4$ arcsec, as verified from observations of several planetary sources. However, $1 / B_{\text {eff }}$ must be replaced by $F_{\text {eff }} / B_{\text {eff }}$ because IRAM data, as stored, are usually not corrected for the forward efficiency $F_{\text {eff }}$ of the telescope. The value of the latter was 0.86 at $230 \mathrm{GHz}$.

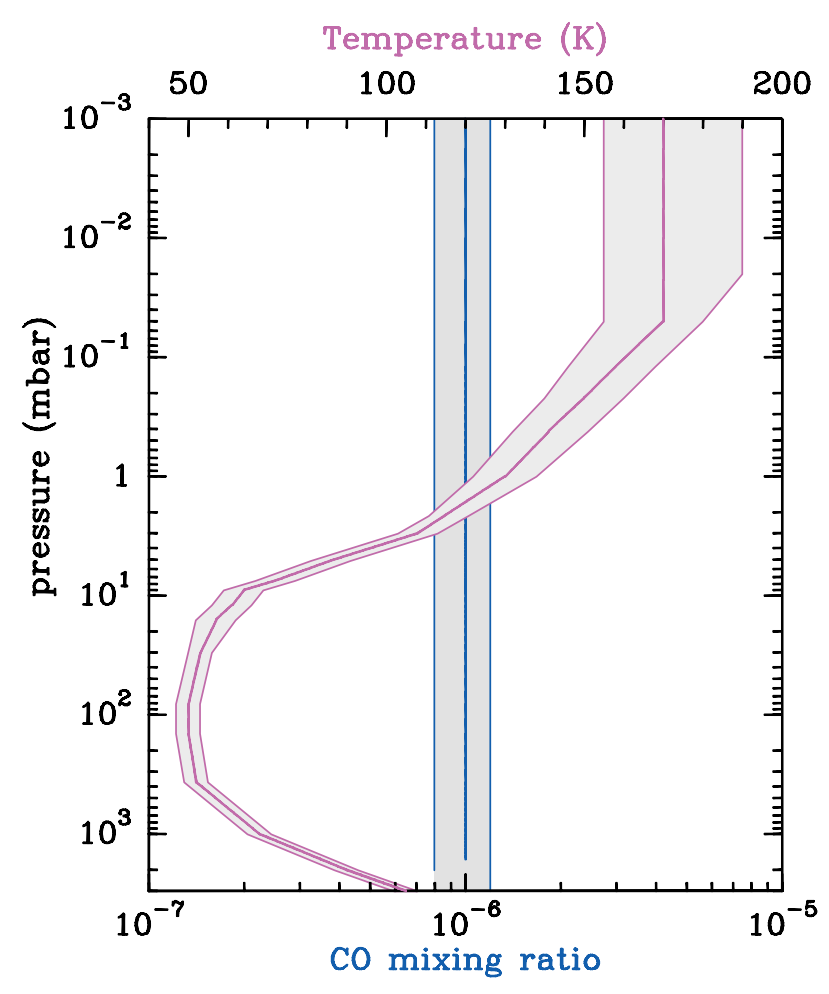

Fig. 5. An atmospheric model of Neptune. The temperature profile and the $\mathrm{CO}$ distribution are determined from the best fits to our $\mathrm{CO}$ spectra (see Fig. 3). The shaded ranges characterize the estimated uncertainties around nominal profiles derived from our data.

A comparison with synthetic calculations of the three $\mathrm{CO}$ emission lines observed on Neptune is presented in Fig. 3. For these preliminary computations we used the thermal profile displayed in Fig. 1 and a mixing ratio of $\mathrm{CO}$ equal to $1 \mathrm{ppm}$, in accordance with all prior CO determinations (see Sect. 1). As noted in Sect. 3 there is good agreement with the data, which underlines the generally good coherence of our $\mathrm{CO}$ dataset. Nevertheless, since we intend to infer the stratospheric thermal profile $T(\mathrm{p})$ of Neptune using a constant-with-height mixing ratio for $\mathrm{CO}$, an in-depth analysis of the $\mathrm{CO}$ spectra is clearly needed. A description of our procedure is given below in Sect. 5.1; the best-fit model, in the least-squares sense, has been determined by trial and error. Uncertainties of $5 \%$ in the absolute $T_{\mathrm{a}}^{*}$ scale, consistent with the data comparisons shown in Fig. 2, will be further considered in the determination of the atmospheric parameters.

\section{Thermal profile of Neptune's atmosphere and its HCN distribution}

\subsection{Retrieval of $T(p)$}

To complement the data shown in Fig. 3 we have used a highresolution spectrum of $\mathrm{CO}(189 \mathrm{kHz})$ obtained at $461 \mathrm{GHz}$ in September 1998, (see Fig. 4). In principle the higher resolution permits us to retrieve useful information at higher altitudes above 0.1 mbar. This expectation originates simply from examination of $\mathrm{CO}$ contribution functions calculated at the lower frequencies in Paper I. We need to note, however, that at this 
higher frequency a severe limitation exists on the atmospheric altitude which can be investigated, since the rotational velocity of Neptune introduces a line broadening of about $3 \mathrm{MHz}$ around the line center, much larger than the effective spectral resolution of these measurements.

The thermal model used as a starting point for our analysis is displayed in Fig. 1. For the stratosphere, at pressure levels above 0.1 mbar, different temperature lapse rates have been adopted by Yelle et al. (1993), Bishop et al. (1998) and Bézard et al. (1999). As first suggested by the former authors, a uniform temperature model was used by Bishop et al. (1998) in the pressure range $0.3-0.001$ mbar with a nominal value of $168 \pm 20 \mathrm{~K}$, while Bézard et al. (1999) indicated a quasiisothermal $(165-180 \mathrm{~K})$ profile in the $(0.02-0.0003 \mathrm{mbar})$ region with a value of $170 \pm 3 \mathrm{~K}$ near $3 \mu \mathrm{bar}$. We thus assumed a constant temperature above $0.01 \mathrm{mbar}$, the lower pressure limit $(<1 \mu \mathrm{bar})$ not being significant since the contribution of the atmosphere above this level is practically negligible and, hence, cannot be measured even with the highest spectral resolution of our data.

One useful supplementary piece of information can be derived from the low spectral resolution data in Fig. 2 for the tropospheric thermal profile. The narrow-band continuum levels measured near the centre frequencies of the $\mathrm{CO} 3-2$ and HCN 4-3 lines (at 345.8 and $354.5 \mathrm{GHz}$ ) show a strong absorption feature of $\mathrm{CO}$ for Neptune and set an important constraint on the background emission at these frequencies.

Taking into account calibration errors of $\pm 5 \%$, a family of distributions $\left(T(\mathrm{p}), q_{\mathrm{CO}}\right)$ has been determined. They correspond to best fits to the three spectral emission lines and the continuum estimates in the extreme wings of the line profiles. For example, since preliminary computations were given in Fig. 3, we show in Fig. 4 a comparison of these improved calculations with our data taken at $461 \mathrm{GHz}$ at the highest spectral resolution. In the line wings the overall shape is the same for both types of spectra recorded in the $J=4-3$ transition, while in the core of the line there are differences depending on whether Neptune's rotation is included or not.

The physical model of Neptune we have inferred from these new calculations is summarized in Fig. 5, where the distribution envelopes characterize the derived $T(\mathrm{p})$ profile as a function of pressure, as well as the uniform distribution $q_{\mathrm{CO}}$. From this model we can conclude that:

1. The best value of $q_{\mathrm{CO}}$ agrees with previous determinations, which lie within the extreme range $0.3-4.5 \times 10^{-6}$ in the stratosphere and troposphere. A complete summary is given by Courtin et al. (1996, see their Fig. 16). Here we propose a mean value of $1.0 \times 10^{-6}$ throughout Neptune's atmosphere, with an estimated error of $20 \%$.

2. Compared with our initial thermal model given in Fig. 1 the present nominal profile is $12 \mathrm{~K}$ warmer in the stratosphere above the $0.1 \mathrm{mbar}$ level and $2 \mathrm{~K}$ colder in the troposphere. This new model increases slightly the CO line contrasts and correctly matches the continuum difference between 354.8 and $354.5 \mathrm{GHz}$ as well as the continuum values measured in the far wings of all $\mathrm{CO}$ lines.

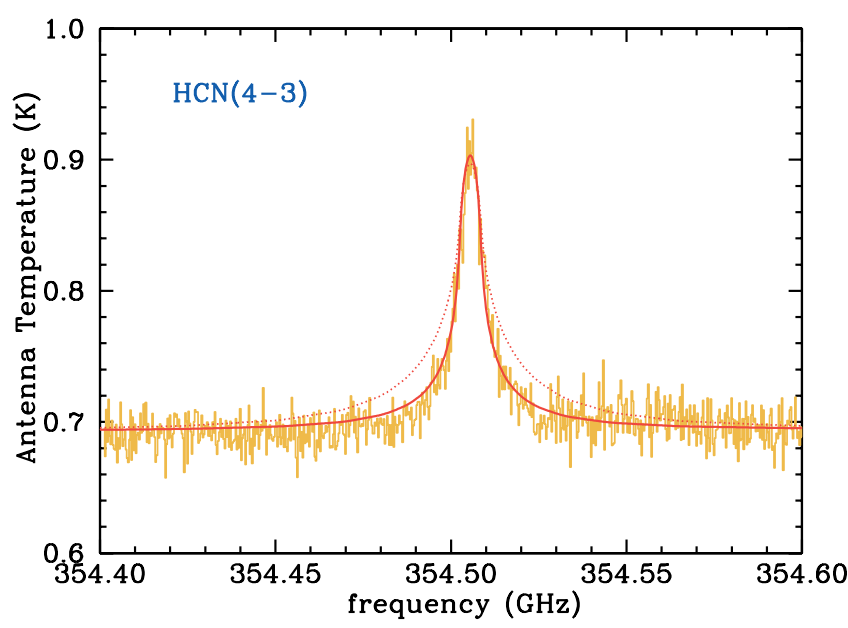

Fig. 6. Antenna temperature spectrum of Neptune observed in the HCN $J=4-3$ rotational transition with a spectral resolution of $378 \mathrm{kHz}$. The total integration time is $200 \mathrm{~min}$, and the spectral coverage is limited to $200 \mathrm{MHz}$. Superposed are two complete calculations using the nominal thermal profile shown in Fig. 5. The solid line corresponds to the best fit model for HCN. The dotted line represents a calculation assuming a mixing ratio of $1.0 \times 10^{-9}$ and the saturation law. Both HCN models are presented in Fig. 7.

3. The temperature structure is close to the nominal diskaveraged profile determined from ISO measurements taken in 1997 (see Bézard et al. 1999). We note, however, that our nominal profile is $8 \mathrm{~K}$ warmer in the pressure range between 0.05 and $1 \mathrm{mbar}$, and also $3 \mathrm{~K}$ colder below $20 \mathrm{mbar}$, than the ISO thermal profile. Based on the present data, the temperature is very uncertain in the upper stratosphere above $\sim 0.03$ mbar.

\subsection{The HCN vertical distribution}

Our HCN $(J=4-3)$ observation is displayed in Fig. 6 together with the line shape corresponding to the nominal temperature profile (see Fig. 5) derived from the $\mathrm{CO}$ observations. The inferred mixing-ratio profile for $\mathrm{HCN}$ is shown in Fig. 7. The dotted curve in Fig. 6 is an HCN spectrum computed for a single mixing ratio of $1.0 \times 10^{-9}$ and the saturation law (see Eq. (1)); it is clearly incompatible with the present data. For consistency with the results presented in Fig. 5 uncertainties are defined by envelope profiles.

Although smaller than that observed in the $\mathrm{CO} 4-3$ transition, the line broadening effect due to the rotation of Neptune is also clearly visible in the HCN 4-3 line profile. As a result, it makes the determination of the HCN profile in the upper atmosphere above 0.01 mbar more uncertain, since the line contrast is substantially decreased and presents small variations with large mixing ratio gradient changes. In the lower stratosphere the modeling errors are lower, since $\mathrm{HCN}$ there exhibits a narrow emission lineshape which is well calibrated in terms of relative antenna temperature. Furthermore, the thermal model is also more precise in this part of the atmosphere. 


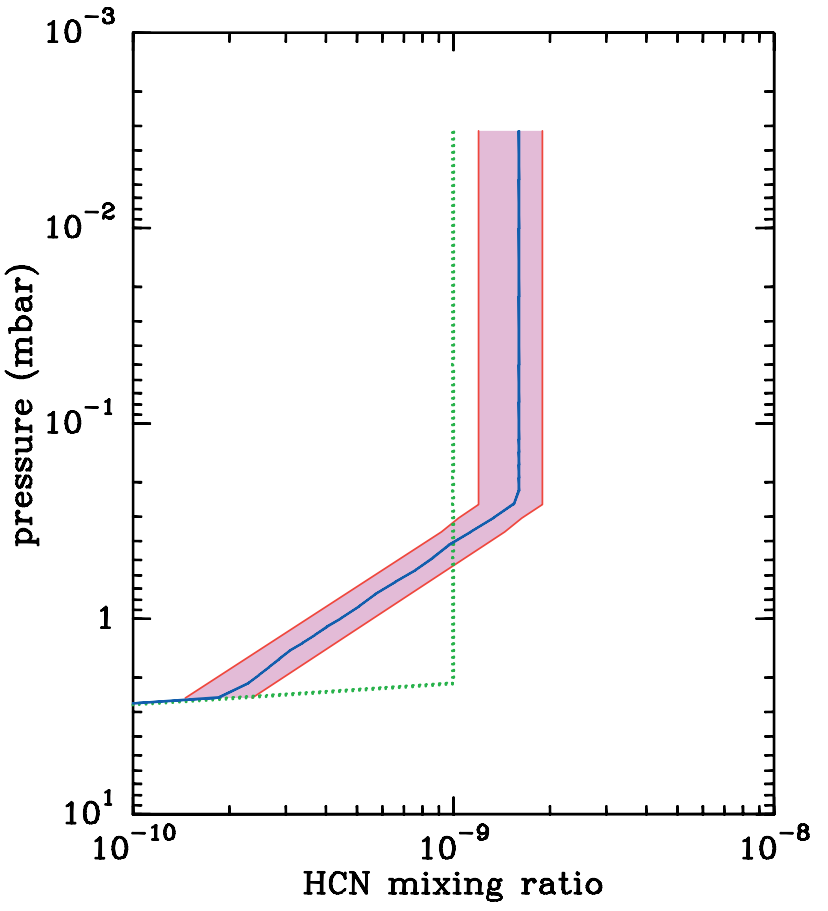

Fig. 7. A model for the HCN mixing ratio in Neptune's upper atmosphere based on the line profile in Fig. 6 and the temperature profile of Neptune's atmosphere derived from our CO observations (solid line). The shaded envelope characterizes the estimated uncertainties around our nominal profile and based on the present dataset. For comparison we show a uniform distribution (dotted curve), limited by the saturation law of $\mathrm{HCN}$ and with the reference mixing ratio of $1.0 \times 10^{-9}$.

\section{Discussion}

On the basis of the observations and analysis presented in this paper it is possible to make a number of comments.

1. The observations are consistent with a uniform distribution of $\mathrm{CO}$ in Neptune's upper atmosphere with a mixing ratio of $1.0 \times 10^{-6}$ within a $20 \%$ uncertainty. We cannot rule out variations in the mixing ratio to lesser degrees, particularly in the troposphere.

The high abundance of $\mathrm{CO}$, and the species' origin deep in Neptune's atmosphere, have been explained by Lodders \& Fegley (1994) as a result of rapid mixing from the deepest levels, where a strong enrichment of $\mathrm{H}_{2} \mathrm{O}$ is expected. Following these authors our upper limit of $\mathrm{CO}$ on Uranus $\left(\sim 10^{-8} \text {, as indicated in Paper } \mathrm{I}\right)^{3}$ is matched also by their updated thermochemical model of the outermost planets. However, as the enrichment factor assumed for $\mathrm{O} / \mathrm{H}$ in the interior of Neptune by Lodders \& Fegley (1994) is very high (440 times solar) and therefore questionable, we suggest that alternate theoretical aspects of $\mathrm{CO}$ formation and dynamics must be plausibly investigated.

Two other oxygen-bearing compounds, $\mathrm{CO}_{2}$ and $\mathrm{H}_{2} \mathrm{O}$, have been identified in the stratosphere of Neptune in ISO

\footnotetext{
3 Encrenaz et al. (2004) have recently demonstrated from infrared spectra that $\mathrm{CO}$ is present in the stratosphere of Uranus with an abundance 30 times lower than that in Neptune.
}

spectra by Feuchtgruber et al. (1997), who have proposed a possible mechanism for the formation of $\mathrm{CO}_{2}$ compatible with the non-detection of this same species on Uranus. In this case $\mathrm{CO}_{2}$ results from the reaction of $\mathrm{CO}$ with $\mathrm{OH}$, the latter radical in turn resulting from the photolysis of $\mathrm{H}_{2} \mathrm{O}$. Even if this formation scheme is not unique (see Feuchtgruber et al. 1997, for details), we note here the importance of $\mathrm{CO}$ as a key component in the formation or absence of some minor compounds on Neptune or Uranus.

2. The nominal tropospheric thermal profile is found to be $2 \mathrm{~K}$ colder than our initial model obtained from the Voyager 2 occultation profile (Lindal 1992). In the region of the tropopause, it is slightly colder than the mean-average equatorial structure observed by Conrath et al. (1998) from Voyager IRIS spectra. Since our observations, and our modeling results, are averages over the whole planetary disk, these temperature discrepancies are not likely to be in significant conflict with the Voyager results.

In the lower stratosphere, the profile appears to be $8 \mathrm{~K}$ warmer between 0.05 and 1 mbar, and $3 \mathrm{~K}$ colder below the 20 mbar level, than the disk-averaged profile proposed by Bézard et al. $(1999,2000)$ to interpret ISO detections of the $\mathrm{CH}_{3}$ radical and $\mathrm{C}_{6} \mathrm{H}_{6}$ on Neptune.

Finally, we note that our modeling applies well for regions into the upper stratosphere (i.e. $~ 0.01$ mbar); above this level, in the mesosphere, the uncertainties become very large.

3. The vertical distribution inferred for $\mathrm{HCN}$ in Neptune is similar in form to that found for Titan (Marten et al. 2002), with an undersaturation at the lowest levels. The relevant column density roughly corresponds to that relative to a constant mixing ratio of $1.0 \times 10^{-9}$ throughout the atmosphere, as estimated from our first detection in 1991. One should note that the latter was obtained with a poorer spectral resolution than in the present paper, and with a modest signal-to-noise ratio.

As initially suggested by Rosenqvist et al. (1992) and Paper I for the formation of HCN on Neptune, detailed numerical models have been described by Lellouch et al. (1994). Two distinct origins, (a) an internal source (due to galactic cosmic ray collisions with $\mathrm{N}$ atoms in Neptune's atmosphere) and (b) an external source of N (via Triton), have been considered, in association with two eddy diffusion coefficient profiles K proposed by Romani et al. (1993). The latter authors developed a one-dimensional photochemical model and used different $\mathrm{K}$ profiles to investigate Voyager observations of hydrocarbons and hazes in the Neptunian stratosphere. A simple first case (A) considers that $\mathrm{K}$ is inversely proportional to the atmospheric number density to some power (typically 0.6 ); the second case (B) exhibits a strong variation at around $1 \mathrm{mbar}$, and presumes a slow, and rapid, mixing in the lower and upper stratospheric regions respectively.

In regard to the $\mathrm{CH}_{3}$ radical present in the upper atmosphere Bézard et al. (1999) found that the case B profile was not appropriate because it yielded too intense a $\mathrm{CH}_{3}$ feature, in disagreement with the ISO observations. As a conservative approach we thus presume that a case A profile is 
more applicable, with a value of $\sim 10^{5} \mathrm{~cm}^{2} \mathrm{~s}^{-1}$ for pressures around 0.1 mbar.

4. Following the results of Lellouch et al. (1994) and adopting a smooth profile for $\mathrm{K}$ (case $\mathrm{A}$, above) we note that the shape of the HCN distribution we have derived is consistent mostly with an internal source of $\mathrm{N}$, and not with that computed for an external source. We caution again however, that our inferred HCN distribution is limited to a stratospheric region located below 0.01 mbar.

We note that our conclusion that elemental nitrogen is necessary for the origin of HCN in Neptune's atmosphere strongly depends on hypotheses of eddy diffusion coefficient profiles and numerical models such as those mentioned above. While awaiting disk-resolved observations of Neptune, the next steps will first require improved predictions of internal and/or external sources of $\mathrm{N}$ atoms on the planet.

Acknowledgements. The James Clerk Maxwell Telescope is operated by the The Joint Astronomy Centre on behalf of the Particle Physics and Astronomy Research Council of the United Kingdom, The Netherlands Organization for Scientific Research, and the National Research Council of Canada. The CSO is operated by the California Institute of Technology under funding from the National Science Foundation. IRAM is supported by INSU/CNRS (France), MPG (Germany) and IGN (Spain). We wish to thank A. Borysow for distributing us her computation programs of $\mathrm{H}_{2}-\mathrm{H}_{2}, \mathrm{H}_{2}-\mathrm{He}$ absorption coefficients.

\section{References}

Bézard, B., Romani, P. N., Conrath, B. J., \& Maguire, W. C. 1991, J. Geophys. Res., 96, 18961

Bézard, B., Romani, P. N., Feuchtgruber, H., \& Encrenaz, Th. 1999, ApJ, 515, 868

Bézard, B., Drossart, P., Encrenaz, Th., \& Feuchtgruber, H. 2000, Icarus, 154, 492

Bishop, J., Romani, P. N., \& Atreya, S. K. 1998, Planet. Space Sci., 46,1

Birnbaum, G., Borysow, A., \& Orton, G. S. 1996, Icarus, 123, 4

Borysow, A., \& Frommhold, L. 1986, ApJ, 304, 849

Burgdof, M., Orton, G. S., Davis, G. R., et al. 2003, Icarus, 164, 244

Conrath, B. J., Flasar, F. M., \& Gierasch, P. J. 1991, J. Geophys. Res., 96, 18931

Conrath, B. J., Gierasch, P. J., \& Ustinov, E. A. 1998, Icarus, 135, 501
Courtin, R., Gautier, D., \& Strobel, D. 1996, Icarus, 123, 37

DeBoer, D. R., \& Steffes, P. G. 1996, Icarus, 123, 324

Draegert, D. A., \& Williams, D. 1968, J. Opt. Soc. Am., 58, 1399

Encrenaz, Th., Serabyn, E., \& Weisstein, E. W. 1996, Icarus, 124, 616

Encrenaz, Th., Lellouch, E., Drossart, P., et al. 2004, A\&A, 413, L5

Feuchtgruber, H., Lellouch, E., De Graauw, T., et al. 1997, Nature, 389,159

Feuchtgruber, H., Lellouch, E., Bézard, B., et al. 1999, A\&A, 341, L17

Flasar, F. M., Conrath, B. J., Gierasch, P. J., \& Pirraglia, J. A. 1987, J. Geophys. Res., 92, 15011

Goldin, A. B., Kowitt, M. S., Cheng, E. S., et al. 1997, ApJ, 488, L161

Griffin, M. J., \& Orton, G. S. 1993, Icarus, 105, 537

Griffin, M. J., Ade, P. A. R., Orton, G. S., et al. 1986, Icarus, 65, 244

Guilloteau, S., Dutrey, A., Marten, A., \& Gautier, D. 1993, A\&A, 279, 661

Hidayat, T., Marten, A., Bézard, B., et al. 1997, Icarus, 126, 170

Lellouch, E., Romani, P. N., \& Rosenqvist, J. 1994, Icarus, 108, 112

Lindal, G. F. 1992, AJ, 103, 967

Lodders, K., \& Fegley, B. Jr. 1994, Icarus, 112, 368

Marley, M. S., \& McKay, C. P. 1999, Icarus, 138, 268

Marten, A., Gautier, D., Owen, T., et al. 1991, IAU Circ., 5531

Marten, A., Gautier, D., Owen, T., et al. 1993a, ApJ, 406, 285

Marten, A., Gautier, D., Matthews, H. E., et al. 1993b, BAAS, 25, 1076

Marten, A., Matthews, H. E., Senay, M., et al. 1995, BAAS, 27, 1088

Marten, A., Hidayat, T., Biraud, Y., \& Moreno, R. 2002, Icarus, 158, 532

Matthews, H. E., Marten, A., Moreno, R., \& Owen, T. 2002, ApJ, 580, 598

Mengel, M., Flatin, D. C., \& De Lucia, F. C. 2000, J. Chem. Phys., 112, 4069

Moreno, R., Marten, A., Biraud, Y., et al. 2001, Planet. Space Sci., 49, 473

Naylor, D. A., Davis, G. R., Griffin, M. J., et al. 1994, A\&A, 291, L51

Pickett, H. M., Poynter, R. L., Cohen, E. A., et al. 1998, J. Quant. Spec. Radiat. Transf., 60, 883

Rohart, F., Derozier, D., \& Legrand, J. 1987, J. Chem. Phys., 87, 5794

Romani, P. N., Bishop, J., Bézard, B., \& Atreya, S. 1993, Icarus, 106, 442

Roques, F., Sicardy, B., French, R. G., et al. 1994, A\&A, 288, 985

Rosenqvist, J., Lellouch, E., Romani, P. N., Paubert, G., \& Encrenaz, Th. 1992, ApJ, 392, L99

Wang, Y., Jaffe, D. T., Graf, U. U., \& Evans, N. J. 1994, ApJS, 95, 503

Weisstein, E. W., \& Serabyn, E. 1996, Icarus, 123, 23

Yelle, R. V., Herbert, F., Sandel, B. R., Vervack, R. J., \& Wentzel, T. M. 1993, Icarus, 104, 38 